\title{
Tracking a Molecular Motor with a Nanoscale Optical Encoder
}

\author{
Charles E. Wickersham, ${ }^{\dagger}$ Kevin J. Cash, ${ }^{\dagger,}$ Shawn H. Pfeil, ${ }^{\dagger, \#}$ Irina Bruck, ${ }^{\perp}$ Daniel L. Kaplan, ${ }^{\perp}$ \\ Kevin W. Plaxco,, ,II and Everett A. Lipman*,†,II
}

${ }^{\dagger}$ Department of Physics ${ }^{\ddagger}$ Department of Chemical Engineering ${ }^{\S}$ Department of Chemistry and Biochemistry, and

"Biomolecular Science and Engineering Program, University of California, Santa Barbara, California 93106

${ }^{\perp}$ Department of Biological Sciences, Vanderbilt University, Nashville, Tennessee 37232

\begin{abstract}
Optical encoders are commonly used in macroscopic machines to make precise measurements of distance and velocity by translating motion into a periodic signal. Here we show how Förster resonance energy transfer can be used to implement this technique at the single-molecule scale. We incorporate a series of acceptor dye molecules into self-assembling DNA, and the periodic signal resulting from unhindered motion of a donor-labeled molecular motor provides nanometer-scale resolution in milliseconds.
\end{abstract}

KEYWORDS Molecular motor, single-molecule, FRET

I n many macroscopic devices, such as desktop printers, disk drives, numerically controlled machine tools, and astronomical telescopes, distance and velocity measurements are made using optical encoders. A typical encoder consists of a light source and sensor, the path between which is occluded by a patterned film having a series of transparent windows. As the source and sensor move with respect to the film, modulation of the sensor output by the windows produces a periodic signal. The distance traveled is then given by the product of the window spacing and the number of periods observed in the signal.

Förster resonance energy transfer ${ }^{1-3}$ (FRET) is a nearfield electromagnetic interaction which, when monitored by photon counting, can be used to measure distances within and between single biomolecules. Two fluorescent dyes, one with relatively short-wavelength absorption and emission (the donor) and the other with longer-wavelength absorption and emission (the acceptor), are attached to the system of interest. A laser is tuned to excite only the donor, which will normally emit a fluorescence photon of its characteristic color within a few nanoseconds. If, however, an acceptor is close by, it can acquire the excitation energy via FRET, and the observed fluorescence will be at a longer wavelength. The probability of transfer (the "energy transfer efficiency") is given approximately by

\footnotetext{
* To whom correspondence should be addressed. E-mail: lipman@physics.ucsb.edu.

I Current address: The Charles Stark Draper Laboratory, Biomedical Engineering Group, 555 Technology Square, Cambridge, Massachusetts 02139.

\# Current address: Pennsylvania Muscle Institute, University of Pennsylvania, Philadelphia, Pennsylvania 19104.

Received for review: 12/18/2009

Published on Web: 02/01/2010
}

$$
E=\frac{1}{1+\left(r / R_{0}\right)^{6}}
$$

where $r$ is the dye separation, and $R_{0}$ (the Förster radius) is a characteristic distance at which $E=0.5$. In typical single-molecule experiments, $R_{0} \approx 5 \mathrm{~nm}$. An estimate of $E$, and hence $r$, can be obtained from the fraction of detected photons emitted by the acceptor. Owing to departures from eq 1 and inherently low signal-to-noise ratios, it is difficult to obtain absolute distances with much better than $10 \%$ precision using single-pair FRET. ${ }^{2,3}$

Molecular motors are essential components of the machinery of life, enabling processes including DNA replication, transcription and repair, protein synthesis, and muscle movement. Single-molecule measurements ${ }^{4-7}$ have yielded a wealth of otherwise inaccessible information about the mechanisms by which these biomolecules move and function. To achieve nanometer-precision tracking of individual motor proteins, available fluorescence techniques rely on long integration times, thousands of fluorophores, or large quantum dot labels. ${ }^{4}$ Magnetic and optical trapping assays ${ }^{5-7}$ require that significant tension be applied to the motor or its substrate. In a 1999 review, ${ }^{1}$ Weiss proposed using periodic arrays of FRET acceptors to monitor digestion of DNA by a single nuclease and transcription by a tethered RNA polymerase. Prior to now, this suggestion had not been realized. In a related ensemble measurement, Yin et al. ${ }^{8}$ recently used sequential quenching to track the average motion of a population of synthetic molecular walkers as they passed three spectrally distinct DNA-bound fluorophores over a period of tens of minutes. Here we describe the synthesis and use of a "FRET encoder," similar to the configuration envisioned by Weiss, that circumvents uncertainties in FRET distance determinations by producing a 
A

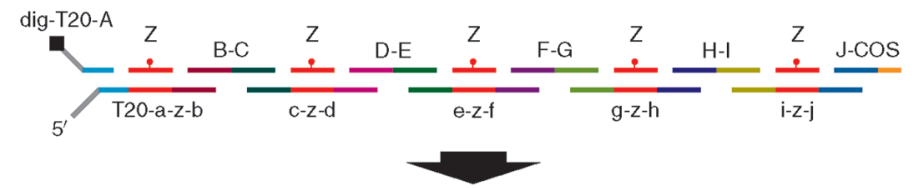

annealing + ligation
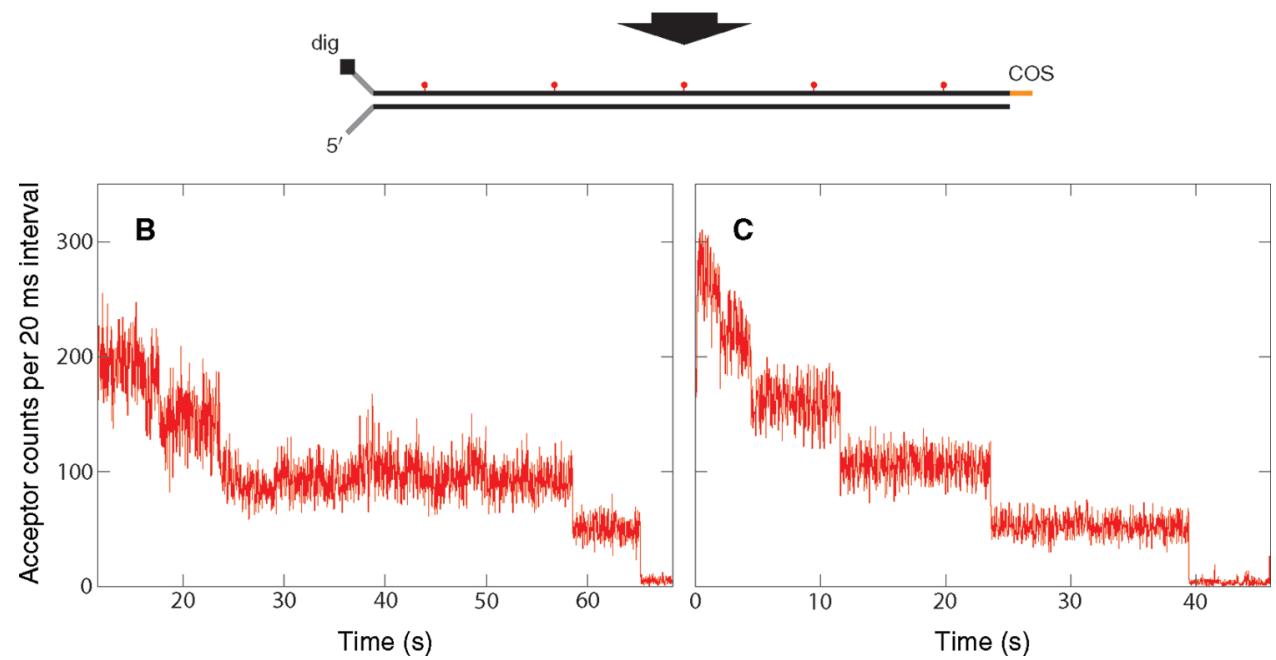

FIGURE 1. Synthesis and verification of FRET encoders. (A) DNA self-assembly scheme for 5-period encoders (see Supporting Information Table 1 for sequences). Each unique sequence is identified with a lower-case letter, and its complement with the corresponding upper-case letter. For example, oligonucleotide E-D (read from $5^{\prime}$ to $3^{\prime}$ ) pairs only with c-Z-d and e-z-f, while e-z-f pairs only with G-F, Z, and E-D. Sequence $\mathrm{Z}$ is internally labeled with an acceptor dye. The free $5^{\prime}$ poly(dT) tail facilitates DnaB loading, while the digoxigenin label at the $3^{\prime}$ terminus allows the encoder to be immobilized by attachment to antidigoxigenin on a fused-silica surface. The $5^{\prime}$ COS overhang is complementary to the 12 bp single-stranded cos site of the $\lambda$ phage genome. (B,C) Sacrificial photobleaching of four- (B) and five-period (C) encoders by direct excitation at $633 \mathrm{~nm}$ verifies complete self-assembly. More than $80 \%$ of tested encoders produced the expected photobleaching pattern.

periodic signal, thereby enabling rapid and precise tracking of single molecular motors.

We synthesized FRET encoders from a set of phosphorylated single-stranded (ss) DNA oligonucleotides. These were designed to form, upon annealing and ligation, doublestranded (ds) DNA labeled with an acceptor fluorophore every 69 base pairs (bp) (see Figure 1, Figure 2 inset, and Supporting Information Table 1). Following synthesis and purification, encoders were ligated at one end to biotinylated $\lambda$ DNA handles. The other end was terminated with two single-stranded poly $(\mathrm{dT})$ tails, one of which was labeled with digoxigenin at the $3^{\prime}$ terminal end. Complete self-assembly was verified by continuously exciting sacrificial encoders with a $1 \mu \mathrm{W} 633 \mathrm{~nm}$ laser, resulting in successive photobleaching of the dyes over a period of tens of seconds (Figure 1). A discrete downward step in the fluorescence data indicates bleaching of a single dye, and we find that the number of photobleaching steps corresponds precisely to the number of dyes designed into the encoders.

To test the FRET encoder, we chose DnaB, the primary replicative helicase from Escherichia coli. ${ }^{9-18}$ Single-molecule techniques have already revealed many properties of helicases, proteins that translocate along DNA, separating the double helix into its component strands. Stochastic behaviors that would be difficult to detect with ensemble methods, such as backtracking, ${ }^{19}$ strand switching, ${ }^{20,21}$ and pausing, ${ }^{19,22}$ have all been observed. Direct application of

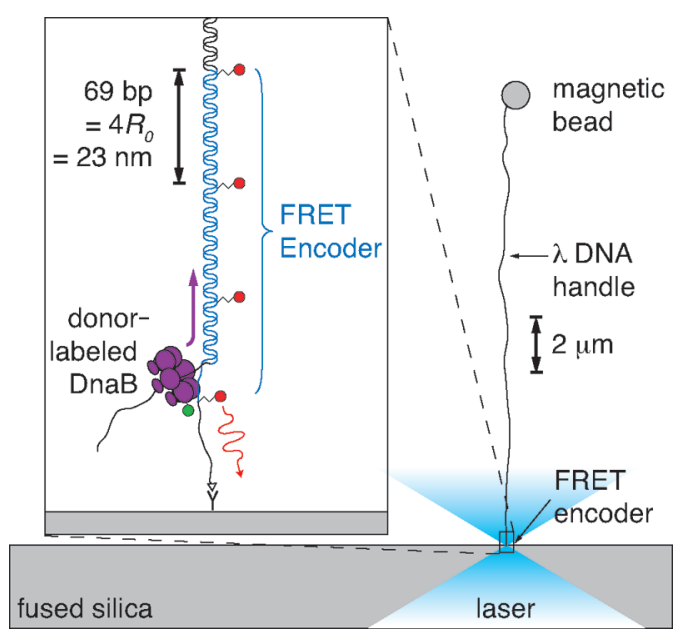

FIGURE 2. Illustration of the experiment, approximately to scale. The FRET encoder is tethered via an antidigoxigenin/digoxigenin linkage to a fused-silica coverslip. A biotinylated $\lambda$ DNA handle, ligated to the untethered end of the encoder, is attached to a streptavidin-coated magnetic bead. A $0.5-3.0 \mathrm{pN}$ vertical magnetic force is applied to the bead, pulling it away from the surface and aligning the encoder with the optical axis. Donor-labeled DnaB helicase diffuses into the focus and loads onto the free $5^{\prime}$ tail of the encoder. As the encoder is unwound, the moving laser-excited donor passes one acceptor after another, inducing long-wavelength fluorescence via FRET. The resulting periodic acceptor signal reports on the motion. A spacing of $4 R_{0}$ between acceptor dyes was chosen so that the donor would be within $2 R_{0}$ of only a single acceptor at any given time. 
force to DNA substrate molecules has been used to differentiate between possible unwinding mechanisms and to examine the role played by ATP. ${ }^{20,22-24}$ In addition, singlemolecule measurements of interactions between helicases and other replication proteins have demonstrated how coordination may arise. ${ }^{25,26}$ Myong et al. ${ }^{27}$ have used singlepair FRET to measure the hepatitis C virus NS3 helicase, proposing a "spring-loaded" unwinding mechanism. Ha and co-workers have also observed "repetitive shuttling" behavior for Escherichia coli Rep helicase along ssDNA, ${ }^{28}$ and strand switching of human BLM helicase. ${ }^{21}$

DnaB is an ATP-driven hexamer that encircles one strand of DNA and translocates in the $5^{\prime}$ to $3^{\prime}$ direction, displacing the nonencircled complementary strand. ${ }^{9}$ It is processive, completing several enzymatic cycles before dissociating from its substrate. ${ }^{18}$ While a number of studies have focused on the structure and function of DnaB ${ }^{9,13,14,16,17}$ and its interaction with other replisome components, ${ }^{10,12,15,26,29,30}$ there have been few direct measurements of the velocity and processivity (distance traveled prior to dissociation) of DnaB alone, ${ }^{12,18}$ more of which will improve our understanding of its unwinding mechanism and movement.

DnaB monomer, expressed and purified as described by Yuzhakov et al., ${ }^{31}$ was sparsely and nonspecifically labeled with an amine-reactive form of the FRET donor dye Alexa Fluor 488 (Invitrogen), and dialyzed exhaustively. For this first experiment, nonspecific labeling provided several advantages: it was not necessary to mutate the protein, there was no possibility that an unfortunate choice of label site would prevent us from collecting data, and we were able to observe a variety of signal forms. It should be noted that a change in label position parallel to the helicase axis would be expected to cause only an overall shift in the phase of the FRET encoder signal. To decrease the likelihood that a single hexamer would carry more than one donor fluorophore, at most $5 \%$ of monomers were labeled.

Using the FRET encoder, we measured donor-labeled DnaB as it unwound DNA (Figure 2). By means of the $3^{\prime}$ digoxigenin labels, FRET encoders were tethered to a fusedsilica coverglass, which formed the bottom wall of a flow cell. A magnetic bead was attached to the distal end of each encoder via the biotinylated $\lambda$ DNA handle. The flow cell was mounted on a combined confocal detection system and magnetic tweezer (Supporting Information Figure 2). A constant force ${ }^{32}$ between 0.5 and $3.0 \mathrm{pN}$ was applied to the magnetic beads, pulling them away from the surface and aligning the encoders parallel to the optical axis.

A buffered solution containing between 32 and $200 \mathrm{nM}$ DnaB hexamer and $5 \mathrm{mM}$ ATP was added to the flow cell, after which a preselected encoder was automatically centered using the detectors (see Supporting Information). A $488 \mathrm{~nm}$ laser, reduced to a power between 11 and $15 \mu \mathrm{W}$, was then focused on the sample in order to excite the FRET donor attached to the helicase.
The resulting acceptor fluorescence data (Figure 3) consist of a series of peaks, with the number of peaks in each complete event correlating precisely with the number of dyes in the corresponding encoder. No events were detected in control experiments without DnaB, nor with unlabeled DnaB. Measurement of the donor signal was precluded by relatively high concentrations of labeled DnaB, required to promote hexamer stability, achieve unwinding activity, ${ }^{11}$ and produce a sufficient number of visible events. At the lowest concentration used in these experiments, there were on average approximately three labeled hexamers per confocal detection volume.

The variation in overall signal level among the Figure 3 events most likely results from suboptimal sample centration and/or focal drift (the risk of encoder photobleaching makes repeated adjustment impractical). Control experiments using a donor-acceptor pair on DNA (data not shown) indicate that with optimal alignment, the maximum count rate is approximately $20 \mathrm{~ms}^{-1}$. Thus a single donor label could account for the signal shown in Figure 3C. Multiple donors on the helicase would be expected to reduce signal contrast and produce additional peaks unless the attachment sites happened to be in close proximity. The gradually increasing background in Figure 3A may have been caused by a growing cluster of acceptor dyes on single-stranded DNA behind the helicase. Because of the time required, it is not possible to calibrate the tension applied to each encoder.

Since donor labeling was not site-specific, and the radius of the DnaB hexamer is approximately equal to $R_{0},{ }^{13}$ the position of the donor (or possibly multiple donors) on the protein could significantly affect the form of the observed signal. In particular, if the label is on the outer surface, then a $180^{\circ}$ rotation of the protein about its central axis could change the donor-acceptor distance by as much as $2 R_{0}$. Figure 3B and C (see also Supporting Information Figure 3D) exhibits modulation of peak heights that could be caused by rotation of the protein with respect to the acceptor-labeled strand, or possibly by multiple donors. A low-frequency envelope may indicate gradual rotation of the helicase, or might instead result from a nonlinear combination, similar to aliased sampling or interfrequency beating, of the oscillations associated with translation and rapid rotation. Future measurements employing longer encoders and site-specific protein labeling will better indicate the causes of the observed modulation.

Consistent correspondence between the maximum number of contiguous periodic peaks and the number of encoder dyes strongly suggests that the observed signals result from translation of DnaB. To separate the primary translational frequency from modulation, noise, and background drift, we computed the power spectra of the four events shown in Figure 3. For each event, the mean count rate was subtracted from all bins to remove the zero-frequency component. The signal was then padded with zeros to eight times its original length, and a discrete Fourier transform was calculated with 

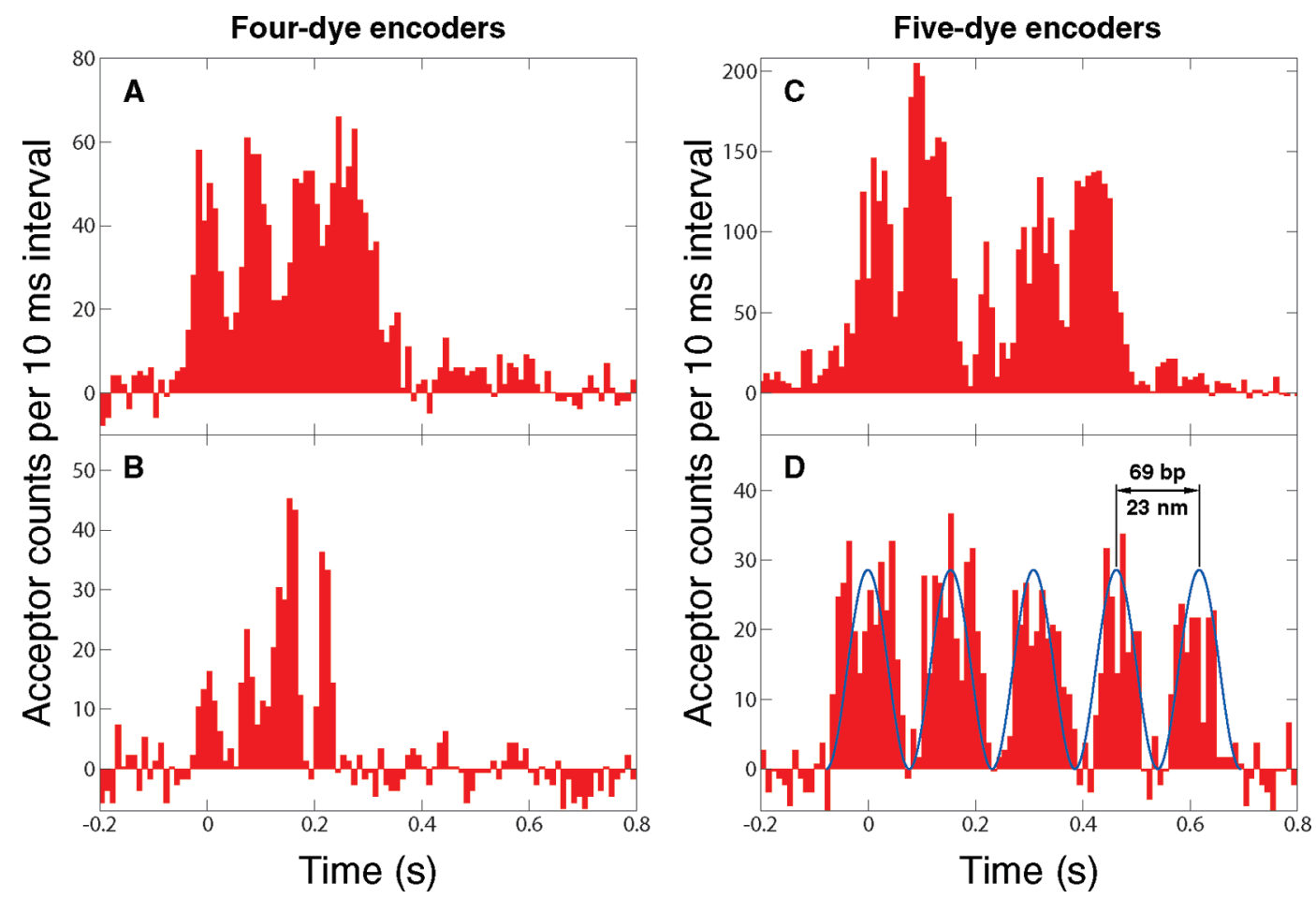

FIGURE 3. Acceptor fluorescence signal as a function of time (background subtracted). A signal peak is produced each time the donor-labeled helicase passes an acceptor dye on its encoder. For each event, the unwinding speed was determined by fitting a function of the form $A \cos ^{2}\left[\pi f\left(t-t_{0}\right)\right]$ to the data using least-squares. One such fit is illustrated in (D). The speeds were (A) $759 \pm 13$, (B) $950 \pm 24$, (C) $665 \pm 12$, and (D) $447 \pm 6 \mathrm{bp} / \mathrm{s}$. A complete event indicates that an individual labeled helicase traveled at least $276 \mathrm{bp}$ (A,B) or $345 \mathrm{bp}$ (C,D). This implies processivity much greater than has previously been measured for DnaB alone.
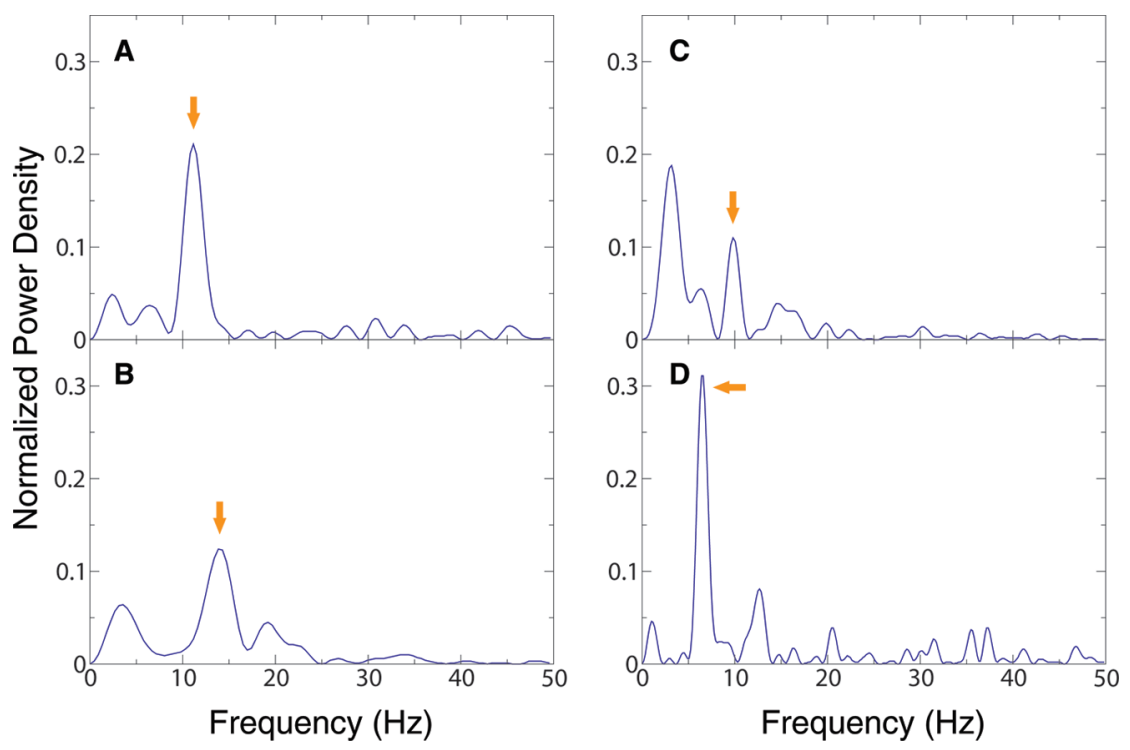

FIGURE 4. Power spectra for the four events shown in Figure 3. The frequency generated by translation of the helicase can be estimated for a complete event by dividing the number of encoder dyes by the event duration. Peaks corresponding to the estimated frequencies are indicated above by arrows. Significant low-frequency components seen in (B) and (C) may be caused by rotational modulation (see text). Side lobes appearing in these power spectra do not exceed one twentieth the height of the associated peaks.

no windowing. Figure 4 shows the squared moduli of the positive-frequency portions of the four transforms.

Since acceptor intensity is determined by eq 1 , the leading and trailing edges of the measured peaks are steep, and the observed signals will not be sinusoidal, even in the absence of modulation. Some power will consequently appear in harmonics above the translational frequency. The finite duration of an event sets a lower bound on its power spectrum peak widths. Widths in excess of this fundamental limit can be caused by nonuniformity of helicase motion (for 
example, a slight slowing can be seen in the third and fourth peaks of Figure 3D) and by the small amount of noise that happens to be in a frequency band near the peak.

Precisely estimating the frequency of a noisy, limitedduration signal is a complicated problem, particularly if the underlying functional form is not known. ${ }^{33,34}$ In addition to locating peaks in the power spectra, we computed leastsquares fits to the acceptor fluorescence data using functions of the form $A \cos ^{2}\left[\pi f\left(t-t_{0}\right)\right]$. One such fit, corresponding to the translational frequency component in the power spectrum, is illustrated in Figure 3D. Frequencies from the fits were multiplied by the $69 \mathrm{bp}$ encoder period to obtain helicase speeds (see Figure 3 caption). Single standard deviation uncertainties were determined from the fit parameters. ${ }^{35}$ For the event shown in Figure 3C, the translational frequency at the power spectrum peak differed by 1.2 standard deviations from that found by fitting. For events A, B, and D, the differences were less than one standard deviation. The largest fractional uncertainty was only $2.5 \%$, indicating that the two frequency estimates are consistent, and that these events exhibit nearly constant-speed unwinding.

For a sample consisting of the events shown in Figure 3 and eight additional events shown in Supporting Information Figure 3, helicase speeds were determined to be between 230 and $1060 \mathrm{bp} / \mathrm{s}$, with a mean of $660 \mathrm{bp} / \mathrm{s}$ and a standard deviation of $250 \mathrm{bp} / \mathrm{s}$. This large variation is consistent with other single-molecule helicase measurements. ${ }^{19}$ The highest unwinding velocities we observe exceed those previously reported in room-temperature ensemble studies of DnaB alone (our measurements were made at $22 \pm 1{ }^{\circ} \mathrm{C}$ ), but are comparable to the average speed of a complete E. coli replication fork in vivo. ${ }^{12,18}$ The speed of DnaB is known to vary over a wide range, ${ }^{36}$ being strongly dependent on interactions with other proteins involved in DNA replication, and on temperature. ${ }^{18}$

A complete five-period event with constant phase advance indicates that a individual labeled helicase has unwound a length of DNA equal to or greater than 345 base pairs (an event begins and ends with the donor approximately half a period from the closest encoder dye). The probability is low that more than one labeled helicase is involved in the production of a single periodic event. The peak intervals in Figure 3 are nearly uniform, exhibiting no pauses for dissociation of one helicase and replacement by another. For more than one labeled helicase to line up and produce a signal with the observed contrast would require that they maintain a fixed spacing close to a multiple of the encoder dye interval ( $69 \mathrm{bp}$ ), with the speeds of the trailing helicases equal to that of the lead, or some unlikely combination of fixed spacing and synchronized, phase-staggered rotational motion. Furthermore, there is no reason to expect a trailing helicase on the separated unlabeled strand to remain in contact with the encoder strand. Additional labeled helicases would also increase the number of peaks per event beyond the number of encoder dyes.
These results contrast significantly with a prior ensemble study reporting a processivity of approximately $10 \mathrm{bp}$ for DnaB. ${ }^{18}$ Tanner et al. $^{26}$ recently found that interactions between DnaB and other replisome components increase its processivity to $10.5 \pm 0.9$ kilobases but did not detect unwinding activity of DnaB alone.

Owing to the form of eq 1, the position resolution of the FRET encoder varies over the course of each cycle, peaking at $r \approx R_{0}$. Using the observed signal and background levels, it is possible to estimate the signal-to-noise ratio $\mathrm{SNR}_{r=R_{0}}$ (see Supporting Information), which will depend on the laser excitation power, the integration time, and the change in dye separation (the step size). For the $4.5 \mathrm{bp}(1.5 \mathrm{~nm})$ translation in a $10 \mathrm{~ms}$ bin of Figure 3D, we expect $\mathrm{SNR}_{r=R_{0}} \approx 2.6$. This is consistent with the abrupt nonrandom transitions seen on the leading and trailing edges of the peaks and compares well with other single-molecule techniques using similar integration times. ${ }^{4-7}$

Under the conditions used to collect the data in Figure $3 \mathrm{D}, \mathrm{SNR}_{r=R_{0}} \approx 1800(P \tau)^{1 / 2}$ for a single base pair $(0.34 \mathrm{~nm})$ step, where $P$ is the laser power in watts and $\tau$ is the measurement time in seconds. Presently, $P=11 \mu \mathrm{W}$ and $\tau=10 \mathrm{~ms}$, giving $\mathrm{SNR}_{r=R_{0}} \approx 0.6$. While this is clearly not adequate to resolve stepping motion, one can envision an experiment on a somewhat slower molecular motor, such as a DNA polymerase, ${ }^{37}$ which allows translocation to be triggered. This would enable the use of much higher excitation power without fear of bleaching the encoders. With $P=200 \mu \mathrm{W}$, we would expect $\mathrm{SNR}_{r=R_{0}} \approx 5$ for a 1 bp step in $40 \mathrm{~ms}$. Thus the prospects appear good for high-resolution FRET encoder measurements of full-speed processive molecular motion.

The technique we have demonstrated could be extended in several directions. We have recently synthesized longer FRET encoders using polymerization and rolling-circle DNA amplification $^{38}$ (to be published elsewhere). These will enable increased precision through signal averaging over many periods. A single-molecule quadrature encoder, which would report both speed and direction by producing two signals of the same frequency with a $\pm \pi / 2$ phase difference, could be synthesized by replacing the acceptor-labeled oligonucleotide with one containing two labels of different colors. Absolute molecular location could be determined using a bicolor FRET encoder with two different acceptor periods, and rotary FRET encoders could be used for measuring flagellar motors and other rotating biomolecular assemblies.

Encoders with vastly improved photostability could be constructed using quantum dots, ${ }^{39,40}$ and rigid encoders fabricated on inorganic substrates could be used to monitor translation, rotation, and flexure of future engineered nanostructures.

Because FRET encoders have high resolution, are independent of force, require no calibration beyond a priori knowledge of acceptor dye spacing, and are potentially applicable to a wide range of molecular motors and other 
nanoscale objects, we expect this technique to find use in many new measurements.

Acknowledgment. Omar Saleh and Noah Ribeck provided us with essential advice and software for using magnetic tweezers, many useful suggestions regarding DnaB helicase, and comments on the manuscript. We thank Megan Valentine and Keir Neuman for comments on the manuscript, Deborah Fygenson for discussions of FRET encoder synthesis, and Benjamin Schuler for advice regarding protein labeling. This work was supported by the Hellman Family Foundation, the Human Frontier Science Program, and the California Nanosystems Institute. K.J.C. and K.W.P. were supported by National Institutes of Health Grant GM062958-01. E.A.L. is an Alfred P. Sloan research fellow.

Supporting Information Available. Detailed methods, DNA sequences, supporting figures and data, data analysis, and signal-to-noise calculation. This material is available free of charge via the Internet at http://pubs.acs.org.

\section{REFERENCES AND NOTES}

(1) Weiss, S. Science 1999, 283, 1676-1683.

(2) Dolghih, E.; Ortiz, W.; Kim, S.; Krueger, B. P.; Krause, J. L.; Roitberg, A. E. J. Phys. Chem. A 2009, 113, 4639-4646.

(3) Camley, B. A.; Brown, F. L. H.; Lipman, E. A.J. Chem. Phys. 2009, 131,104509

(4) Park, H.; Toprak, E.; Selvin, P. R. Q. Rev. Biophys. 2007, 40, $87-$ 111

(5) Greenleaf, W. J.; Woodside, M. T.; Block, S. M. Annu. Rev. Biophys. Biomol. Struct. 2007, 36, 171-190.

(6) Moffitt, J. R.; Chemla, Y. R.; Smith, S. B.; Bustamante, C. Annu Rev. Biochem. 2008, 77, 205-228.

(7) Neuman, K. C.; Nagy, A. Nat. Methods 2008, 5, 491-505.

(8) Yin, P.; Choi, H. M. T.; Calvert, C. R.; Pierce, N. A. Nature 2008, 451, 318-322.

(9) LeBowitz, J. H.; McMacken, R. J. Biol. Chem. 1986, 261, 47384748.

(10) Mok, M.; Marians, K. J.J. Biol. Chem. 1987, 262, 16644-16654

(11) Bujalowski, W.; Klonowska, M. M.; Jezewska, M. J. J. Biol. Chem. 1994, 269, 31350-31358

(12) Kim, S. S.; Dallmann, H. G.; McHenry, C. S.; Marians, K. J. Cell 1996, 84, 643-650.

(13) San Martin, C.; Radermacher, M.; Wolpensinger, B.; Engel, A.; Miles, C. S.; Dixon, N. E.; Carazo, J. M. Structure 1998, 6, 501 509
(14) Jezewska, M. J.; Rajendran, S.; Bujalowska, D.; Bujalowski, W. J. Biol. Chem. 1998, 273, 10515-10529

(15) Barcena, M.; Ruiz, T.; Donate, L. E.; Brown, S. E.; Dixon, N. E.; Radermacher, W.; Carazo, J. M. EMBO J. 2001, 20, 1462-1468.

(16) Kaplan, D. L.; O’Donnell, M. Mol. Cell 2002, 10, 647-657.

(17) Yang, S. X.; Yu, X. O.; VanLoock, M. S.; Jezewska, M. J.; Bujalowski, W.; Egelman, E. H. J. Mol. Biol. 2002, 321, 839-849.

(18) Galletto, R.; Jezewska, M. J.; Bujalowski, W.J. Mol. Biol. 2004, 343, 83-99.

(19) Perkins, T. T.; Li, H. W.; Dalal, R. V.; Gelles, J.; Block, S. M. Biophys. J. 2004, 86, 1640-1648.

(20) Dessinges, M. N.; Lionnet, T.; Xi, X. G.; Bensimon, D.; Croquette, V. Proc. Natl. Acad. Sci. U.S.A. 2004, 101, 6439-6444.

(21) Yodh, J. G.; Stevens, B. C.; Kanagaraj, R.; Janscak, P.; Ha, T. EMBO J. 2009, 28, 405-416.

(22) Dumont, S.; Cheng, W.; Serebrov, V.; Beran, R. K.; Tinoco, I.; Pyle, A. M.; Bustamante, C. Nature 2006, 439, 105-108.

(23) Lionnet, T.; Spiering, M. M.; Benkovic, S. J.; Bensimon, D.; Croquette, V. Proc. Natl. Acad. Sci. U.S.A. 2007, 104, 1979019795.

(24) Johnson, D. S.; Bai, L.; Smith, B. Y.; Patel, S. S.; Wang, M. D. Cell 2007, 129, 1299-1309.

(25) Lee, J. B.; Hite, R. K.; Hamdan, S. M.; Xie, X. S.; Richardson, C. C.; van Oijen, A. M. Nature 2006, 439, 621-624.

(26) Tanner, N. A.; Hamdan, S. M.; Jergic, S.; Schaeffer, P. M.; Dixon, N. E.; van Oijen, A. M. Nat. Struct. Biol. 2008, 15, 170-176.

(27) Myong, S.; Bruno, M. M.; Pyle, A. M.; Ha, T. Science 2007, 317, 513-516.

(28) Myong, S.; Rasnik, I.; Joo, C.; Lohman, T. M.; Ha, T. Nature 2005, $437,1321-1325$

(29) Fang, L. H.; Davey, M. J.; O’Donnell, M. Mol. Cell 1999, 4, 541 553.

(30) Schaeffer, P. M.; Headlam, M. J.; Dixon, N. E. IUBMB Life 2005, $57,5-12$

(31) Yuzhakov, A.; Turner, J.; O’Donnell, M. Cell 1996, 86, 877-886

(32) Gosse, C.; Croquette, V. Biophys. J. 2002, 82, 3314-3329.

(33) Kay, S. M. Modern Spectral Estimation: Theory and Application; Prentice Hall: New York, 1988

(34) Quinn, B. G.; Hannan, E. J. The Estimation and Tracking of Frequency; Cambridge University Press: New York, 2001

(35) Wolberg, J. Data Analysis Using the Method of Least Squares; Springer Verlag: New York, 2006.

(36) Indiani, C.; Langston, L. D.; Yurieva, O.; Goodman, M. F.; O’Donnell, M. Proc. Natl. Acad. Sci. U.S.A. 2009, 106, 6031-6038.

(37) Christian, T. D.; Romano, L. J.; Rueda, D. Proc. Natl. Acad. Sci. U.S.A. 2009, 106, 21109-21114

(38) Beyer, S.; Nickels, P.; Simmel, F. C. Nano Lett. 2005, 5, 719-722.

(39) Michalet, X.; Pinaud, F. F.; Bentolila, L. A.; Tsay, J. M.; Doose, S.; Li, J. J.; Sundaresan, G.; Wu, A. M.; Gambhir, S. S.; Weiss, S. Science 2005, 307, 538-544.

(40) Yin, Y.; Alivisatos, A. P. Nature 2005, 437, 664-670. 


\title{
Supporting Information for
}

\section{Tracking a Molecular Motor with a Nanoscale Optical Encoder}

\author{
Charles E. Wickersham, ${ }^{1}$ Kevin J. Cash, ${ }^{2,6}$ Shawn H. Pfeil, ${ }^{1,7}$ Irina Bruck, ${ }^{5}$ \\ Daniel L. Kaplan, ${ }^{5}$ Kevin W. Plaxco, ${ }^{3,4}$ and Everett A. Lipman ${ }^{1,4}$ \\ ${ }^{1}$ Department of Physics \\ ${ }^{2}$ Department of Chemical Engineering \\ ${ }^{3}$ Department of Chemistry and Biochemistry \\ ${ }^{4}$ Biomolecular Science and Engineering Program \\ University of California, Santa Barbara, California 93106, USA \\ ${ }^{5}$ Department of Biological Sciences, Vanderbilt University, Nashville, Tennessee 37232, USA \\ ${ }^{6}$ Present address: The Charles Stark Draper Laboratory, Biomedical Engineering Group, \\ 555 Technology Square, Cambridge, Massachusetts, 02139, USA \\ ${ }^{7}$ Present address: Pennsylvania Muscle Institute, University of Pennsylvania, \\ Philadelphia, Pennsylvania 19104, USA
}

\section{Supporting Methods}

Buffers and Reagents. All solutions were prepared with distilled deionized water in order to minimize fluorescence background.

Protein labeling (see below) was carried out in a buffer consisting of $0.15 \mathrm{M}$ sodium bicarbonate and $1 \mathrm{mM}$ sodium azide, adjusted to $\mathrm{pH} 8.3$ with $\mathrm{HCl}$ ("reaction buffer"). Labeled DnaB helicase was stored in $20 \%$ w/v glycerol, $20 \mathrm{mM}$ Tris, and $500 \mu \mathrm{M}$ EDTA, adjusted to $\mathrm{pH} 7.5$ with $\mathrm{HCl}$ ("storage buffer").

DNA was stored in $10 \mathrm{mM}$ Tris- $\mathrm{HCl}(\mathrm{pH} 8.0)$ with 1 mM EDTA ("TE buffer").

The buffer used for DnaB helicase measurements ("helicase buffer") was $50 \mathrm{mM}$ Tris adjusted to $\mathrm{pH} 7.9$ at room temperature with $\mathrm{HCl}, 10 \mathrm{mM}$ magnesium acetate, $0.1 \mathrm{mg} / \mathrm{ml}$ bovine serum albumin (Sigma-Aldrich B4287), $100 \mu \mathrm{M}$ EDTA (Sigma-Aldrich E7889), ${ }^{1}$ and $0.01 \% \mathrm{w} / \mathrm{v}$ Pluronic F127 surfactant (Sigma-Aldrich P2443).

An enzymatic oxygen-scavenging system, consisting of $35 \mu \mathrm{g} / \mathrm{ml}$ catalase (Sigma-Aldrich C1345), $0.2 \mathrm{mg} / \mathrm{ml}$ glucose oxidase (Sigma-Aldrich G7141), $4.5 \mathrm{mg} / \mathrm{ml} \beta$-D-glucose (Sigma-Aldrich G8270), and $2 \mathrm{mM}$ Trolox antioxidant (Sigma-Aldrich 238813), ${ }^{2}$ was used during fluorescence measurements.
The flow cell passivation solution was TE buffer containing 1\% w/v Pluronic F127.

DNA Sequences. All unlabeled single-stranded DNA sequences were purchased from Integrated DNA Technologies (Coralville, IA), as was the Cy5 version of the labeled "Z" oligonucleotide (see main text Fig. 1A and Supporting Table 1). The $\mathrm{Z}$ sequence containing Alexa Fluor 594 was purchased from IBA Biologics GmbH (St. Louis, MO).

FRET Encoder Synthesis. FRET encoders were designed to self-assemble from a set of unique singlestranded oligonucleotide components (Supporting Table 1), which were combined in TE buffer at an equal molar ratio, except for $\mathrm{Z}$, which was added at $40 \times$ or $50 \times$ molar excess for the four- or five-period encoders, respectively. The component mixture was heated to $75^{\circ} \mathrm{C}$ for 10 minutes and then cooled over approximately 1 hour to $37{ }^{\circ} \mathrm{C}$. The temperature was held at $37{ }^{\circ} \mathrm{C}$ for three days to allow the encoders to fully anneal. Singlestranded nicks between oligomers were ligated with T4 DNA ligase (New England Biolabs M0202S) according to manufacturer instructions. Encoders were purified on a $1.6 \% \mathrm{w} / \mathrm{v}$ agarose gel and ligated to biotinylated $\lambda$ phage genomic DNA molecules (New England Bio- 
labs N3011S). The full-length product was finally purified with a spin column (Invitrogen K3100-01) according to manufacturer instructions. Proper self-assembly of the encoders was verified by gel electrophoresis (Supporting Fig. 1) and by photobleaching assays (main text

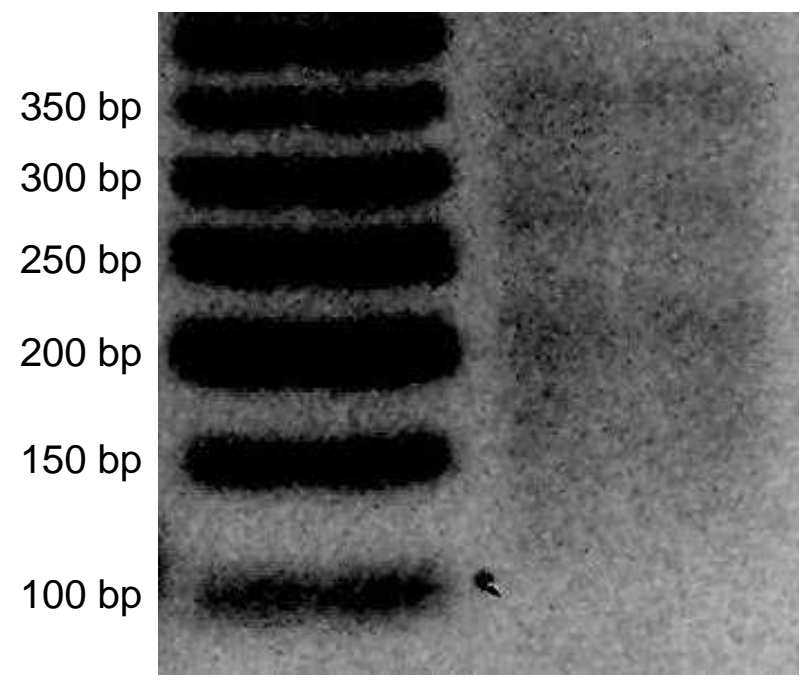

Supporting Figure 1. Gel electrophoresis separation of a 50 bp ladder (left lane, New England Biolabs N3236S) and the products of a four-period FRET encoder synthesis. The band separation in the right lane corresponds to addition of a single $69 \mathrm{bp}$ encoder period. The full-length $276 \mathrm{bp}$ product migrates at the same rate as a 350 bp blunt-ended DNA duplex because of the two 20-nucleotide poly(dT) tails (main text Fig. 1A). A third lane (not shown) containing the ligated FRET encoder was run on this gel, but was shielded from UV light in order to prevent DNA damage. Encoders for use in single-molecule experiments were purified by excising the area in the third lane next to the slowest band in the FRET encoder lane shown above.

Fig. 1). The encoder structure supports in vitro reconstitution of DnaB-catalyzed unwinding: the helicase loads on the free $5^{\prime}$ tail, and the $3^{\prime}$ tail of the acceptor-labeled strand is forced around the periphery of the hexamer. ${ }^{3}$

DnaB Labeling. In order to remove Tris prior to aminereactive labeling, $10 \mu \mathrm{L}$ of $15 \mu \mathrm{M}$ DnaB monomer stock solution was dialyzed against $2 \mathrm{~L}$ of reaction buffer using 12-14 kDa cutoff regenerated cellulose tubing (Fisher Scientific 21-152-8). $2 \mu \mathrm{L}$ of $75 \mu \mathrm{M}$ Alexa Fluor 488 carboxylic acid, succinimidyl ester (Invitrogen A20000) was then added to the DnaB solution and allowed to react for 24 hours. The completed reaction was dialyzed against $1 \mathrm{~L}$ of reaction buffer to remove reactive Alexa Fluor 488, followed by dialysis against $2 \mathrm{~L}$ of storage buffer. Recovery of protein was $50 \%$, with at most $5 \%$ of monomers labeled, as determined by UV and visible absorbance. All steps were conducted at $4{ }^{\circ} \mathrm{C}$. As the label is amine reactive, the dye was not site-specifically attached to the DnaB monomers.

Flow Cell Preparation. Flow cells were fabricated using two coverslips, a $25 \mathrm{~mm}$ fused-silica square (Esco Products R425025) and an $18 \mathrm{~mm}$ Pyrex square. The coverslips were bonded together with two strips of paraffin wax/polyolefin film (Parafilm M, Alcan Packaging), which formed a flow channel 1-2 mm wide with a volume of approximately $5 \mu \mathrm{L}$.

Prior to bonding, coverslips were sonicated in $2 \%$ v/v Micro-90 glass cleaning solution (Cole-Parmer EW18100-01) for 10 minutes, and then in distilled deionized water for 10 minutes. They were blown dry with filtered compressed air, exposed to a hand-held corona surface treater (Electro-Technic Products BD-20AC) for $10 \mathrm{sec}-$ onds, and vapor-coated in a sealed Petri dish with a drop of hexamethyldisilazane for 20 minutes. The Parafilm strips were placed between the coverslips and melted under pressure on a $120^{\circ} \mathrm{C}$ hotplate until a reliable seal was formed.*

A solution containing $10 \mathrm{pM}$ encoder DNA in TE buffer and $1 \mathrm{nM}$ anti-digoxigenin was prepared, allowing anti-digoxigenin to bind to digoxigenin labels on the $3^{\prime}$ termini of the encoders (main text Fig. 1A). This solution was placed in the flow cell for 30 minutes so that antidigoxigenin would adsorb to the fused silica surface, after which the cell was flushed with $50 \mu \mathrm{L}$ of passivation solution. Fluid exchange was accomplished by drawing old solution out of the flow cell with absorbent paper. After 10 minutes, $2 \mu \mathrm{L}$ of passivation solution containing $100 \mathrm{pM}$ of $1 \mu \mathrm{m}$-diameter streptavidin-coated superparamagnetic beads (Invitrogen 650-01) were added to the flow cell. The beads were allowed to bind to the biotinylated $\lambda$ DNA handles for 10 minutes, after which unbound beads were washed out of the cell with $100 \mu \mathrm{L}$ of passivation solution.

In preparation for fluorescence measurements, the cell was flushed with $10 \mu \mathrm{L}$ of oxygen scavenging sys-

${ }^{*}$ After the experiments, fused-silica coverslips were recovered by soaking flow cells in hexane overnight to dissolve the Parafilm. The coverslips were then submerged for 30 minutes in a mixture of 4 parts by volume $17.8 \mathrm{M}$ sulfuric acid to one part $30 \%$ w/w hydrogen peroxide to remove any organic residue. 


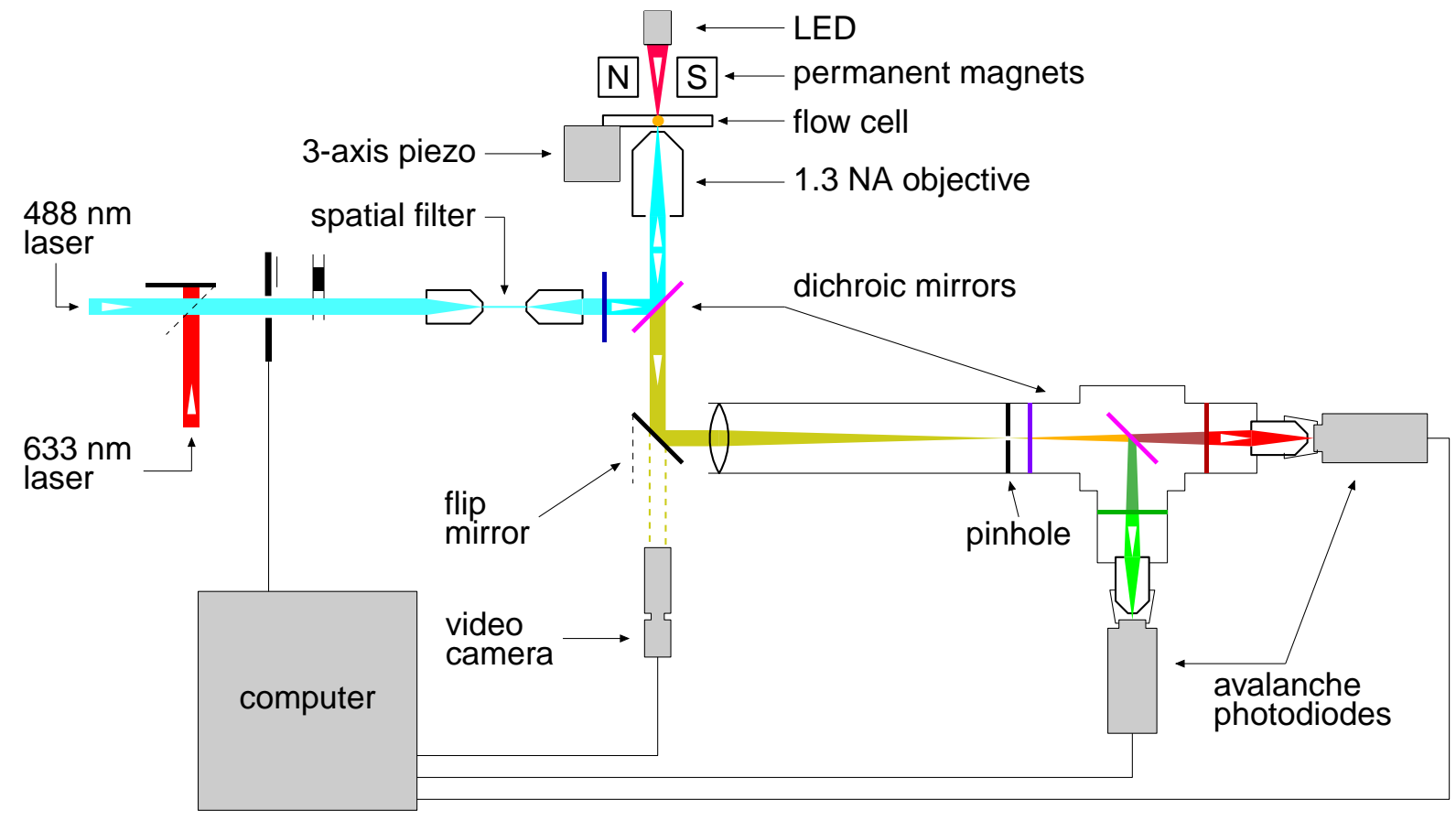

Supporting Figure 2. Custom confocal microscope and magnetic tweezer apparatus used for single-molecule fluorescence measurements.

Supporting Table 1. Single-stranded oligonucleotide sequences for FRET encoders. All except T20-a-z-b have a $5^{\prime}$ phosphate to facilitate ligation. Sequence A-T20 has a 3' digoxigenin label. Two different versions of sequence Z were prepared, each internally labeled with a FRET acceptor dye between bases 12 and 13. The version used in the four-period encoder contained Alexa Fluor 594, while the five-period encoder used Cy5. When paired with the donor dye Alexa Fluor 488, Alexa Fluor 594 and Cy5 produce similar Förster radii $\left(R_{0}\right)$ between 5 and $6 \mathrm{~nm}$. The five-period encoder uses all sequences except COS-H. The four-period encoder uses all sequences except for COS-J, i-z-j and I-H.

\begin{tabular}{|c|l|}
\hline Name & Nucleotide Sequence $\left(\mathbf{5}^{\prime} \rightarrow \mathbf{3}^{\prime}\right)$ \\
\hline $\mathbf{Z}$ & GTCTTGGATCGTTCTGCATATTG \\
COS-H & AGGTCGCCGCCGTGTCCATGTTCCAACTCCTTGC \\
COS-J & AGGTCGCCGCCCGTGTATCATTGTGGCACTCGGAG \\
A-T20 & GATCACTGAGTACGTGTTTTTTTTTTTTTTTTTT \\
C-B & CTCGTTAGCGCGAATCGATGACCTTATTATCCACGGTAAGGAATGT \\
E-D & AACTTGGAAGCTTCGCGCACCAATACAGCAAGAACCGTGCGGTAAC \\
G- $-\mathrm{f}$ & CGGAGCTGTGCAGGTGTCACGGCTCACCGGCTCAATCGATATCAAG \\
I-H & TTAACCGTATCACGCCAGGAACGGTGTCCATGTTCCAACTCCTTGC \\
c-z-d & GGTCATCGATTCGCGCTAACGAGCAATATGCAGAACGATCCAAGACGTTACCGCACGGTTCTTGCTGTA \\
e-z-f & TTGGTGCGCGAAGCTTCCAAGTTCAATATGCAGAACGATCCAAGACCTTGATATCGATTGAGCCGGTGA \\
g-z-h & GCCGTGACACCTGCACAGCTCCGCAATATGCAGAACGATCCAAGACGCAAGGAGTTGGAACATGGACAC \\
i-z-j & CGTTCCTGGCGTGATACGGTTAACAATATGCAGAACGATCCAAGACCTCCGAGTGCCACAATGATACAC \\
T20-a-z-b & TTTTTTTTTTTTTTTTTTTCACGTACTCAGTGATCCAATATGCAGAACGATCCAAGACACATTCCTTACCGTGGATAATAA \\
\hline
\end{tabular}


tem in helicase buffer.

Fluorescence Measurements. Fluorescence measurements were carried out using the custom-built confocal microscope $\mathrm{e}^{4,5}$ and magnetic tweezer apparatus illustrated in Supporting Fig. 2. One of two CW laser excitation sources can be selected with a flip mirror, at $488 \mathrm{~nm}$ (Coherent Sapphire 488-25) or $633 \mathrm{~nm}$ (JDS Uniphase 1507P-1). The excitation beam is spatially filtered, after which it is brought to focus by a 1.3 numerical aperture, $100 \times$ oil-immersion microscope objective (Olympus UPLFLN 100XO2). The flow cell is mounted in a custom holder attached to a 3-axis feedback-controlled piezoelectric stage (Physik Instrumente P-611.3S). The cell is oriented so that observations are made through the fused-silica coverslip.

Fluorescence emission is collected by the excitation objective, separated from scattered laser light by a dichroic mirror (Chroma Z488/633RPC), and focused through a $100 \mu \mathrm{m}$-diameter pinhole. After additional laser light rejection by a long-pass filter (Omega Optical 493AELP), donor and acceptor fluorescence photons are separated by a second dichroic mirror (Omega Optical 560DCLP), bandpass filtered (Chroma Technology D525/50m and D630/60m for the donor and acceptor channels, respectively), and detected by avalanche photodiodes (PerkinElmer Optoelectronics SPCM-AQR16). Photon arrival times are recorded by a $100 \mathrm{ps}$ time digitizer (Ortec 9353) and stored on a computer.

Magnetic beads (see above) attached to sample molecules can be manipulated with a pair of $\mathrm{NdFeB}$ magnets (Dura Magnetics NS-505050) mounted on linear and rotary translation stages. The 3-D position of each bead can be tracked in real time at $60 \mathrm{~Hz}$ by imaging its interference pattern on a CCD camera (Basler scA640-70fm).

An assembled flow cell was mounted in the singlemolecule instrument and prepared as described above. A field of view, typically containing on the order of 10 beads, was then located using the camera. The magnetic beads were pulled away from the surface with a force ${ }^{6}$ of $0.5-3.0 \mathrm{pN}$, which is small enough to avoid significant alteration of protein-DNA interactions. ${ }^{7}$

Occasionally, beads will bind to multiple tethers, or individual tethers will adhere non-specifically to the glass surface. In order to identify potential problems of this nature, the magnetic tweezers were rotated 600 times, causing multiple and rotationally-constrained tethers to coil, thereby reducing the heights of the affected beads. At the end of this procedure, beads which were less than the expected distance from the fusedsilica surface were eliminated from consideration. Several encoders under the remaining beads were located as described below and excited directly with $1 \mu \mathrm{W}$ at $633 \mathrm{~nm}$ in order to verify complete assembly by stepwise photobleaching (see main text Fig. 1).

The maximum length of the encoders, about $120 \mathrm{~nm}$, is approximately an order of magnitude smaller than the axial extent of the excitation and detection volumes of the optical system. Proper centering of the sample therefore ensures that the rates of excitation and detection remain constant along the length of the encoder. The equal heights of the observed photobleaching steps confirm that the vertical position of the focal volume was optimized.

A solution was prepared containing $5 \mu \mathrm{L}$ of $100 \mathrm{mM}$ ATP and $95 \mu \mathrm{L}$ of oxygen scavenging system in helicase buffer. The flow cell was flushed with approximately $10 \mu \mathrm{L}$ of this solution, after which DnaB helicase was thawed and added to the remainder. For each batch of $\mathrm{DnaB}$, the concentration was adjusted based on labeling efficiency so that there would be approximately $10 \mathrm{nM}$ of labeled monomer in solution. The required hexamer concentrations were between 32 and $200 \mathrm{nM}$.

A bead was selected and illuminated from above by an LED. The confocal volume was positioned near the centroid of the resulting diffraction pattern, and focused at the cover slip surface. The LED was then turned off, the acceptor-channel avalanche photodiode was turned on, and the DnaB/ATP solution described above was introduced into the flow cell. The $633 \mathrm{~nm}$ laser, reduced to $1 \mu \mathrm{W}$ with absorptive neutral density filters, was used for direct excitation of the acceptor dyes while the sample stage was scanned in an outwardly-directed spiral search pattern. When a significant signal was detected, an algorithm previously described by Ha et al. ${ }^{8}$ was used to precisely center the confocal volume on the FRET encoder, after which the beam shutter was immediately closed to minimize photobleaching.

The $488 \mathrm{~nm}$ laser, reduced to a power between 11 and $15 \mu \mathrm{W}$, was selected as the excitation source, and the shutter was reopened. Fluorescence photon arrival times were recorded for approximately one minute, after which a new encoder was found and the process repeated. This was necessary because direct excitation of acceptor dyes by the $488 \mathrm{~nm}$ laser caused them to pho- 
tobleach after a few minutes. ${ }^{9}$ The acceptor signal from direct excitation at $488 \mathrm{~nm}$ is, however, negligible. Significant fluorescence in the acceptor channel indicates FRET from a nearby labeled helicase.

The event frequency was very low, and consequently hours of observation were required to collect a single complete periodic signal. Helicase concentration could not be increased, as leakage into the acceptor channel of fluorescence from donor-labeled DnaB in solution was the dominant source of background noise.

\section{Supporting Discussion}

Data Analysis. Acceptor photon arrival times were binned in $10 \mathrm{~ms}$ intervals to produce a fluorescence intensity time series. Periodic events with sufficient signalto-noise ratios, such as those shown in main text Fig. 3 and Supporting Fig. 3, were readily apparent from visual inspection of the data. Once an event was located, its start time was determined by approaching in the direction of increasing time until a bin was found with a signal level at least one third of the maximum for that event. The event was then scanned from this bin in the direction of decreasing time, until a bin was found at no more than one fourth the maximum level, and that point was designated the start time. The end time was found in a similar manner, with the initial scan in the direction of decreasing time, from a point after the event. For events $\mathrm{D}$ and $\mathrm{H}$ in Supporting Fig. 3, thresholds were adjusted to compensate for large variations in peak height. Helicase translation speeds were determined as described in the main text.

Additional Events. Additional five-period encoder events are shown in Supporting Fig. 3. Direct excitation of acceptors by the $488 \mathrm{~nm}$ laser is infrequent, but does occur. This effect and FRET can both eventually cause photobleaching of encoder dyes. It is therefore natural to expect that some events will have fewer peaks than the number of acceptors on the encoder. Bleaching of the donor is also possible, and would result in the abrupt loss of acceptor signal. Furthermore, if the helicase encounters a nick in either DNA backbone, it will fall off of or shear the encoder, and quickly diffuse away. Supporting
Figs. 3B and $3 \mathrm{C}$ show examples of events in which the second of five encoder dyes appears to be missing, and the events in Supporting Figs. 3F and 3G also show only four peaks. In Supporting Fig. 3H, the signal disappears in the middle of the last peak. Shortly after that event was detected, the number of active acceptors remaining on the encoder was checked by switching to direct excitation with the $633 \mathrm{~nm}$ laser. The fluorescence count rate was then approximately $80 \%$ of its original value. This measurement strongly suggests that of the possible causes of an incomplete event, acceptor photobleaching is the most likely in this case.

Events with fewer than two distinct peaks cannot be distinguished from occasional scattering caused by contaminants drifting through the laser focus.

Resolution. The observed acceptor fluorescence signal in a FRET encoder measurement is directly proportional to the energy transfer efficiency $E$. Owing to the form of main text Eq. 1, the position resolution varies over the course of each encoder period. From that equation, we find

$$
\frac{d E}{d r}=\frac{-6 r^{5}}{R_{0}^{6}\left[1+\left(r / R_{0}\right)^{6}\right]^{2}} .
$$

At a signal maximum, when the donor-acceptor separation $r \approx 0, d E / d r \approx 0$, and no signal change results from incremental movement of the donor. On the leading and trailing edges of the signal peaks where $r=R_{0}$, however, $|d E / d r|=3 / 2 R_{0}$. At $r=R_{0}=5 \mathrm{~nm}$, a displacement of $1 \mathrm{bp}(0.34 \mathrm{~nm})$ will then result in a change from $E=0.45$ to $E=0.55$, producing a proportional increase in acceptor signal. ${ }^{*}$

In a single measurement of duration $\tau$, for example one $10 \mathrm{~ms}$ bin in main text Fig. 3D, we collect a signal $S=E A$, where $A$ is the maximum observed signal. ${ }^{\dagger} A$ is a function of laser power $P$ and of $\tau$. So long as $P$ is kept below approximately $200 \mu \mathrm{W}$ in our system, saturation of the dyes will not occur, and $A=a P \tau$, where $a$ is a constant. We then have

$$
S=E a P \tau .
$$

For a small change $\Delta r$, the corresponding signal change

$$
\Delta S \approx \frac{d S}{d r} \Delta r=\frac{d S}{d E} \frac{d E}{d r} \Delta r=a P \tau \frac{d E}{d r} \Delta r .
$$
time.

${ }^{*}$ The $4 R_{0}$ acceptor spacing of the present encoders prevents significant interaction between the donor and more than one acceptor at a

${ }^{\dagger}$ Here we assume $E \approx 1$ at the point in the encoder cycle when the donor and acceptor are closest, and that the difference between a molecular motor step and the corresponding change in $r$ is negligible. 

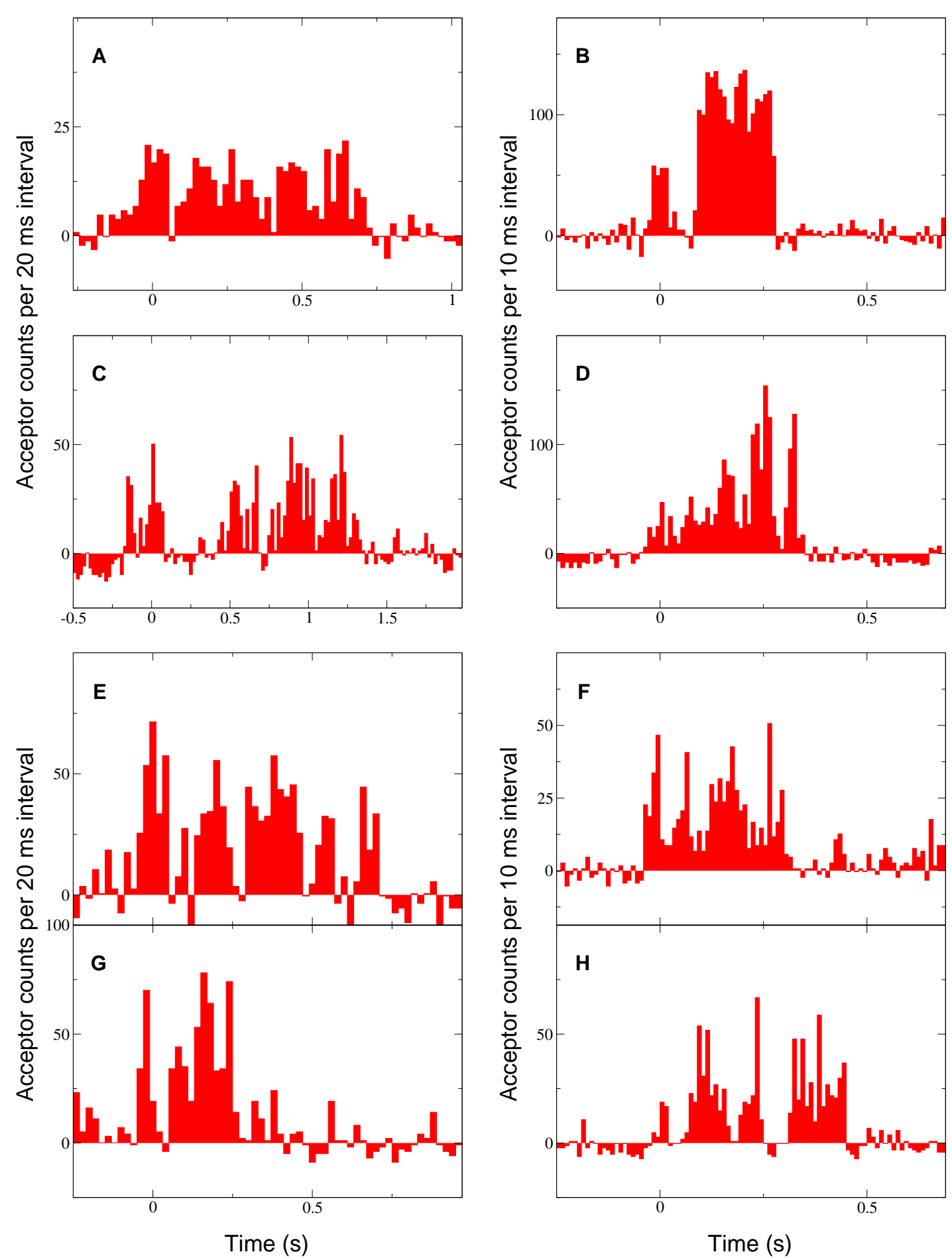

Supporting Figure 3. Additional five-period encoder events. Helicase translation speeds were determined by fitting functions of the form $A \cos ^{2}\left[\pi f\left(t-t_{0}\right)\right]$ to the data using least squares. The speeds were (A) $432 \pm 7 \mathrm{bp} / \mathrm{s}$, (B) $1060 \pm 30 \mathrm{bp} / \mathrm{s}$, (C) $229 \pm 5 \mathrm{bp} / \mathrm{s}$, (D) $850 \pm 18 \mathrm{bp} / \mathrm{s}$, (E) $390 \pm 11 \mathrm{bp} / \mathrm{s}$, (F) $700 \pm 20 \mathrm{bp} / \mathrm{s}$, (G) $790 \pm 20 \mathrm{bp} / \mathrm{s}$, and (H) $610 \pm 16 \mathrm{bp} / \mathrm{s}$. For all events except (B), speeds determined using power spectra were within $4 \%$ of those given here. A missing peak and poor signal contrast in (B) prevented reliable frequency determination using the power spectrum. 
FRET encoder measurements contain both shot noise and fluorescence background. The shot noise $\sigma_{s}=\sqrt{S}$. As with $A$, the background level $B=b P \tau$, where $b$ is constant. After background subtraction, a noise component $\sigma_{B}=\sqrt{B}$ remains. Since shot noise and background noise are uncorrelated, the total noise

$$
n=\sqrt{\sigma_{s}^{2}+\sigma_{B}^{2}}=\sqrt{S+B}=\sqrt{(E a+b) P \tau} .
$$

It follows that for a change in separation $\Delta r$, the signal-to-noise ratio

$$
\begin{aligned}
\mathrm{SNR} & \equiv \frac{|\Delta S|}{n}=\frac{a P \tau}{\sqrt{(E a+b) P \tau}}\left|\frac{d E}{d r} \Delta r\right| \\
& =\frac{a \sqrt{P \tau}}{\sqrt{E a+b}}\left|\frac{d E}{d r} \Delta r\right| .
\end{aligned}
$$

When $r=R_{0}, E=0.5$, and

$$
\left|\frac{d E}{d r} \Delta r\right|=\frac{3}{2} \frac{|\Delta r|}{R_{0}} .
$$

We then have*

$$
\mathrm{SNR}_{r=R_{0}}=\frac{3 a}{2 \sqrt{a / 2+b}} \sqrt{P \tau} \frac{|\Delta r|}{R_{0}} .
$$

In main text Fig. 3D, the background level $B=$ 16 counts in $\tau=10 \mathrm{~ms}$, and the maximum signal $A \approx 33$ counts. The laser power $P=11 \mu \mathrm{W}$. Thus we find $a=3.0 \times 10^{8} \mathrm{~W}^{-1} \cdot \mathrm{s}^{-1}$, and $b=1.5 \times 10^{8} \mathrm{~W}^{-1} \cdot \mathrm{s}^{-1}$. From Eq. 8,

$$
\mathrm{SNR}_{r=R_{0}}=2.6 \times 10^{4} \sqrt{P \tau} \frac{|\Delta r|}{R_{0}},
$$

with $P$ in watts and $\tau$ in seconds. During that event, the helicase was moving $4.5 \mathrm{bp}(1.5 \mathrm{~nm})$ in $10 \mathrm{~ms}$, so from Eq. 9 we expect $\mathrm{SNR}_{r=R_{0}} \approx 2.6$ in a single bin. For $\Delta r=1 \mathrm{bp}(0.34 \mathrm{~nm})$ and $R_{0}=5 \mathrm{~nm}$, we would have

$$
\operatorname{SNR}_{r=R_{0}}(1 \mathrm{bp})=1800 \sqrt{P \tau} \text {. }
$$

As discussed in the main text, a significant increase in $P$ should be possible in future experiments. Further gains might be realized with near-infrared acceptor dyes, which would have increased spectral separation from the donor. This would reduce background noise from donor photons leaking into the acceptor channel. Since $R_{0}$ depends on the spectral overlap of donor emission and acceptor absorption, it too would be reduced, increasing the SNR.

\section{Supporting References}

[1] Kaplan, D. L. \& O’Donnell, M. DnaB drives DNA branch migration and dislodges proteins while encircling two DNA strands. Mol. Cell 10, 647-657 (2002).

[2] Rasnik, I., McKinney, S. A. \& Ha, T. Nonblinking and long-lasting single-molecule fluorescence imaging. Nature Methods 3, 891-893 (2006).

[3] LeBowitz, J. H. \& McMacken, R. The Escherichiacoli DnaB replication protein is a DNA helicase. $J$. Biol. Chem. 261, 4738-4748 (1986).

[4] Schuler, B., Lipman, E. A. \& Eaton, W. A. Probing the free-energy surface for protein folding with single-molecule fluorescence spectroscopy. Nature 419, 743-747 (2002).

[5] Schuler, B., Lipman, E. A., Steinbach, P. J., Kumke, M. \& Eaton, W. A. Polyproline and the "spectroscopic ruler" revisited with single-molecule fluorescence. Proc. Natl. Acad. Sci. U. S. A. 102, 2754 2759 (2005).

[6] Gosse, C. \& Croquette, V. Magnetic tweezers: Micromanipulation and force measurement at the molecular level. Biophys. J. 82, 3314-3329 (2002).

[7] Bustamante, C., Smith, S. B., Liphardt, J. \& Smith, D. Single-molecule studies of DNA mechanics. Curr. Opin. Struct. Biol. 10, 279-285 (2000).

[8] Ha, T., Chemla, D. S., Enderle, T. \& Weiss, S. Single molecule spectroscopy with automated positioning. Appl. Phys. Lett. 70, 782-784 (1997).

[9] Kong, X. X., Nir, E., Hamadani, K. \& Weiss, S. Photobleaching pathways in single-molecule FRET experiments. J. Am. Chem. Soc. 129, 4643-4654 (2007).

${ }^{*}$ Neither $\left|\frac{d E}{d r}\right|$ nor the SNR given in Eq. 6 peak exactly at $r=R_{0}$. For the purposes of this calculation, however, the difference between the peak value and $\mathrm{SNR}_{r=R_{0}}$ is negligible. For our parameters, the peak SNR occurs at $0.988 R_{0}$, and its value is $0.13 \%$ larger than $\mathrm{SNR}_{r=R_{0}}$. 